\title{
REPRODUCCIÓN Y PRODUCCIÓN DE LECHE DE VACAS CON DISTINTA PROPORCIÓN DE GENES BOS TAURUS
}

\author{
REPRODUCTIVE AND MILK YIELD PERFORMANCE OF CROSSBRED COWS WITH \\ DIFFERENT PROPORTIONS OF BOS TAURUS GENES
}

\author{
López-Ordaz, R.. ${ }^{1 *}$, C. Vite-Cristóbal' ${ }^{1}$ J.G. García-Muñiz y P.A. Martínez-Hernández¹
}

${ }^{1}$ Posgrado en Producción Animal. Departamento de Zootecnia. Universidad Autónoma Chapingo. Km. 38.5 Carretera México-Texcoco. Chapingo. México. 56230.

*rlopezor@yahoo.com; rlopez@correo.chapingo.mx

\section{Palabras ClaVe ADICIONALES}

Bovinos de doble propósito. Cruzamiento. Complementación.

\section{RESUMEN}

El objetivo del presente estudio fue evaluar los factores ambientales y la proporción óptima de genes Bos taurus (BT) y su influencia en la producción de leche (PL) y el comportamiento reproductivo de bovinos cruzados $3 / 4$ Holstein $(\mathrm{H})$ $\times 1 / 4$ Cebú (C); $3 \mathrm{H} 1 \mathrm{C}, 3 / 4$ Suizo (S) $\times 1 / 4 \mathrm{C} ; 3 \mathrm{~S} 1 \mathrm{C}, 1 /$ $2 \mathrm{H} \times 1 / 2 \mathrm{C} ; \mathrm{HC}$ y $1 / 2 \mathrm{~S} \times 1 / 2 \mathrm{C}$; SC. La información analizada se obtuvo de 276 lactancias de 69 vacas de los grupos raciales de tres hatos en Veracruz, México. Las variables estudiadas incluyeron la PL por lactancia (PTL), por vaca/día (PLD), por día de intervalo entre partos (PLIEP), al pico de lactancia (PLMax), días al pico de lactancia (TPLMax), duración de la lactancia (DL), edad al primer parto (EPP), días abiertos (DA) y el intervalo entre partos (IEP). Los resultados obtenidos indicaron que el grupo racial influyó únicamente $(p<0,05)$ la PLT y DL; las vacas $3 \mathrm{H} 1 \mathrm{C}$ produjeron 12,12 , y $34 \%$ más leche por lactancia que los grupos raciales $3 \mathrm{~S} 1 \mathrm{C}, \mathrm{HC}$ y SC, respectivamente. Los animales HC mostraron menores $(p<0,01)$ EPP, DA e IEP que las $3 \mathrm{H} 1 \mathrm{C}, 3 \mathrm{~S} 1 \mathrm{C}$ y SC. Análogamente, las vacas alcanzaron su valor máximo de PTL (4859 kg), TPLMax (39,6 días), DL $(376,8)$, DA (180, 7 días) e IEP (463,5 días) cuando la proporción de genes BT fue $75 \%$. En conclusión, las vacas $3 \mathrm{H} 1 \mathrm{C}$ mostraron mejor PTL y comportamientos moderados en DL, DA e IEP en comparación con los otros grupos raciales. Las animales $\mathrm{HC}$ mostraron mayor habilidad reproductiva que las vacas con genes de Suizo. Los grupos racia-

Recibido: 28-10-06. Aceptado: 22-10-08.

\section{Additional KeYWORDS}

Dual-purpose cattle. Crossbreeding. Supplementation.

les con $75 \%$ de genes BT fueron los más apropiados para producción de leche en el norte de Veracruz.

\section{SUMMARY}

The objective of this study was to evaluate the effect of environmental factors, and to determine the optimum proportion of Bos taurus (BT) genes on milk yield (MY) and reproductive traits of $3 / 4$ Holstein (H) x 1/4 Zebú (Z), 3H1Z; 3/4 Swiss (S) $x$ $1 / 4 Z, 3 S 1 Z ; 1 / 2 \mathrm{H} \times 1 / 2 \mathrm{Z}, \mathrm{HZ}$; y $1 / 2 \mathrm{~S} \times 1 / 2 \mathrm{Z}$, SZ; crossbred cows. Lactation records $(n=276)$ of 69 cows in three commercial dual-purpose farms of Veracruz, Mexico, were used. Total MY (TMY), daily MY (DMY), calving interval MY (CIMY), MY at lactation peak (PMY), days to peak MY (DPMY), lactation length (LL), age at-first calving (AFC), days open (DO), and calving interval $(\mathrm{Cl})$, were analyzed. The obtained results showed that the racial group affected $(p<0.05)$ TMY and LL solely; cows $3 \mathrm{H} 1 \mathrm{Z}$ did produce 12,12 , and $34 \%$ more milk than $3 \mathrm{~S} 1 \mathrm{Z}, \mathrm{HZ}$ and $\mathrm{SZ}$, respectively. Likewise, $\mathrm{HZ}$ cows had better reproductive performance $(p<0.05)$ than $3 \mathrm{H} 1 \mathrm{Z}, 3 \mathrm{~S} 1 \mathrm{Z}$, and $\mathrm{SZ}$, with earlier $\mathrm{AFC}$, and shorter $\mathrm{DO}$ and $\mathrm{Cl}$. In addition, cows did reach maximum TMY (4859 kg), DPMY (39.6 d), LL (376.8 d), DO (180.7 d) and $\mathrm{Cl}(463.4 \mathrm{~d})$ when the proportion of Bos taurus gene was $75 \%$. In conclusion, cows $3 \mathrm{H} 1 \mathrm{Z}$ fed tropical forages and supplemented with $18 \% \mathrm{CP}$ concentrates had 
better performance in TMY and moderate LL, DO and $\mathrm{Cl}$ than $3 \mathrm{~S} 1 \mathrm{Z}, \mathrm{HZ}$ and SZ. Holstein $\mathrm{x}$ Zebu crossbreeding cows had higher reproductive ability than those with genes of Swiss. Results from this study suggest crossbred cows with $75 \%$ BT genes as the most appropriate to produce milk in the tropical conditions of Northern Veracruz.

\section{INTRODUCCIÓN}

Una de las alternativas para mejorar la PL en los trópicos puede ser la utilización de razas nativas Bos indicus (BI) en cruzamiento con razas lecheras Bos taurus (BT). La hipótesis anterior fue confirmada en varios estudios previos (McDowell et al., 1996; Osorio-Arce y Segura-Correa, 2002). En otro estudio, Acharya (1987) observó que las vacas $1 / 2 \mathrm{BT} \mathrm{x} 1 / 2 \mathrm{BI}$ comparadas con las razas locales produjeron $265 \%$ más de leche, consumen más alimento, tuvieron partos tres meses antes, y redujeron en dos o tres veces el periodo seco.

El Norte de Veracruz utiliza 1,6 millones de bovinos que producen 233,07 millones de litros de leche en una superficie de 1,6 millones de ha (INEGI, 2005). En la última década, los SDP de dicha zona manifestaron tendencias a la especialización en la producción de leche (PL). Para lograr dichos objetivos no sólo se requieren animales adaptados a los trópicos, sino también mejorar el pastoreo intensivo del pastoreo, complementación con concentrados y crianza artificial de los terneros (Vite-Cristóbal et al., 2007). Además, los SDP dependen de la efectividad de los cruzamientos y de la habilidad de las vacas para cosechar sus alimentos. Al respecto, hay avances importantes en el manejo de los animales que consumen forrajes nativos, complementados con proteína y energía y su relación con PL; sin embargo, la información relacionada con la evaluación de grupos raciales cruzados BT $\mathrm{x} \mathrm{BI}$, que consumen forrajes tropicales, suplementados con concentrados requiere de estudios más detallados. Con base a lo anterior, los objetivos del presente estudio fue- ron: evaluar el efecto de factores ambientales y determinar la proporción óptima de genes BT en la PL y el comportamiento reproductivo de bovinos cruzados $3 / 4$ Holstein $(\mathrm{H}) \times 1 / 4$ Cebú(C); $3 \mathrm{H} 1 \mathrm{C}, 3 / 4 \operatorname{Suizo}(\mathrm{S})$ $\mathrm{x} 1 / 4 \mathrm{C} ; 3 \mathrm{~S} 1 \mathrm{C}, 1 / 2 \mathrm{Hx} 1 / 2 \mathrm{C}$; $\mathrm{HC} \mathrm{y}^{1} / 2 \mathrm{~S} \mathrm{x} 1 / 2 \mathrm{C}$; $\mathrm{SC}$, que consumen gramíneas tropicales complementadas con concentrados.

\section{MATERIAL Y MÉTODOS}

\section{ANIMALES YMANEJO}

Los datos fueron obtenidos de enero de 1997 a diciembre de 2004 con 276 lactancias completas de vacas $3 \mathrm{H} 1 \mathrm{C}, 3 \mathrm{~S} 1 \mathrm{C}$, HC y SC. Las lactancias tuvieron una duración de 270 días y provinieron de 69 animales de cada grupo racial de tres ranchos ganaderos en Ozuluama, Veracruz. El municipio se localiza a $21^{\circ} 40^{\prime}, 97^{\circ} 51^{\prime} y$ tiene un altitud de $150 \mathrm{~m}$. García (1988) clasificó el clima de la zona como $\mathrm{Aw}_{2}(\mathrm{e}) \mathrm{w}^{\prime}$, con temperatura media anual de $24,9^{\circ} \mathrm{C}$ y precipitación anual de $1074,3 \mathrm{~mm}$. El aporte de genes BI provino de animales Brahman (B), Indobrasil (I) y Gir (G); mientras que los BT fueron de las razas Holstein (H) y Suizo Pardo (S). Las proporciones: $1 / 4,1 / 2,5 / 8$ y $3 / 4$ de genes BT fueron incluidos como $25,50,62,5$ y $75 \%$, respectivamente, además, esas mismas proporciones se usaron como covariable en los modelos de análisis.

Los tres hatos tenían 98, 94 y 99 vacas adultas en producción de cada grupo racial, con la adición de alimento balanceado durante el ordeño, e inseminación artificial (IA). Los hatos fueron seleccionados de 13 ranchos y cada grupo tiene más de ocho generaciones de selección y apareamiento inter se de $1 / 2$ Holstein $\mathrm{x} 1 / 2$ Brahman, $1 / 2$ Suizo $\mathrm{X} 1 / 2$ Brahman, $1 / 2$ Holstein o Suizo $\mathrm{x}$ Indobrasil o Gir. La distribución por grupos raciales fue similar para cada rancho. Los registros productivos con menos de 270 días en leche y las vacas de grupos raciales diferentes a los indicados anteriormente no fueron considerados en el análisis final.

Las vacas se ordeñaban de 6:00 a 8:00 y 
de 17:00 a 19:00 h. El manejo nutricional de los animales consistió en el pastoreo rotacional de gramíneas tropicales. La rotación de los animales en los potreros y el suministro de concentrados se realizaron diariamente. El alimento concentrado comercial ofrecido fue de $18 \%$ de proteína y 1,65 Mcal de energía neta de lactancia (ENL). La asignación de concentrado fue corregida semanalmente con base en la producción individual, asignando $1,0 \mathrm{~kg}$ por cada $3,0 \mathrm{~kg}$ de leche producida en el momento del registro. En los tres ranchos, la base de la alimentación fue el pastoreo de pangola (Digitaria decumbens), señal (Brachiaria decumbens cv. Señal), bermuda (Cynodon dactylon), taiwán (Pennisetum purpureum Schum var. Taiwán) y gramas nativas (Paspalum spp. y Axonopus spp.). El manejo reproductivo de los hatos consistió en empadre continuo e IA. Las actividades reproductivas y la PL se registraron en forma individual diariamente.

Los registros individuales de PL y eventos reproductivos de cada vaca fueron utilizadas para generar las variables: PL por lactancia (PLT), por día (PLD), por día de intervalo entre partos (PLIEP) y al pico de lactancia (PLMax), tiempo al pico de lactancia (TPLMax), duración de la lactancia (DL), edad al primer parto (EPP), días abiertos (DA) y el intervalo entre partos (IEP). La PLT se estimó con el método de interpolación del ICAR (ICAR, 1995); PLD fue el cociente de PLT entre DL; PLIEP el cociente de PLT entre IEP; mientras que PLMax y TPLMax fueron estimados mediante la ecuación de Wood (Schaeffer et al., 1977). La última ecuación se utilizó debido a su mejor ajuste con la prueba de Friedman y sus comparaciones con las ecuaciones de Sikka (1950) y Wood (1967). La DL fue el intervalo en días entre las fechas de parto y secado; la EPP fue la edad en meses al momento del primer parto; el IPC se estimó como el intervalo en días entre las fechas del parto próximo anterior y la de servicio efectivo, y el IEP fue el intervalo en días entre partos consecutivos.

\section{AnÁlisisestadístico}

La información del número de lactancias se agrupó en cuatro categorías, incluyendo animales desde una hasta 10 lactancias. La categoría 2000 incluyó la información de 1997 a 2000, la 2001 y 2002 incluyó la información de los mismos años, mientras que la 2003 incluyó información correspondiente al 2003 más la de 2004. El año de parto también se agrupó en cuatro categorías: 2000, 2001, 2002 y 2003; la primera contuvo los partos de 1997 al 2000, la segunda y la tercera incluyeron información relativa a los años, 2001 y 2002, y la última consideró los años 2003 y 2004. Paralelamente, la información relativa a DA y al IEP fueron ordenadas en dos categorías correspondientes al año de parto: 2000 y 2001; la primera incluyó los partos de 1997 al 2000 y la segunda, los de 2001 al 2004.

Las épocas de parto y de nacimiento se agruparon en lluvias y seca. La precipitación promedio mensual superior a $60 \mathrm{~mm}$ (García, 1988) fue usada para definir las épocas de lluvia; la precipitación inferior a dicho valor fue usada para definir la estación seca del año; con base a este criterio, la época de lluvia fue de junio a octubre, con $83 \%$ de distribución; mientras que la seca fue de noviembre a mayo, con una distribución del 17\% de la precipitación promedio anual.

La PTL, PLD, PLIEP, PLMax, TPLMax, y DL se analizaron con el procedimiento MIXED de SAS (2002). El modelo incluyó los efectos fijos de grupo racial, el número de lactancia, el año de parto y los efectos aleatorios de rancho y vaca anidada en grupo racial y rancho. Las medias de cuadrados mínimos para los efectos principales y las interacciones se obtuvieron con LSMEANS. La opción/DDFM=SATTERTH se usó para calcular los grados de libertad aproximados para los factores que no tuvieron prueba exacta. La prueba de Tukey se utilizó para la comparación de medias de cuadrados mínimos. Las interacciones no 


\section{LÓPEZ-ORDAZ, VITE-CRISTÓBAL, GARCÍA-MUÑIZ, MARTÍNEZ-HERNÁNDEZ}

significativas $(\mathrm{p}>0,05)$ fueron removidas del modelo:

$$
Y_{i j k l m n o}=\mu+R_{i}+G_{j}+L_{k}+A P_{1}+E P_{m}+V(R G)_{n}+e_{i j k l m n o}
$$

Donde:

$\mathrm{Y}_{\mathrm{ijk} \mid m n o}=$ Cada una de las variables de respuesta modeladas,

$\mu=$ Media común para todas las observaciones,

$\mathrm{R}_{\mathrm{i}}=$ Efecto aleatorio del i-ésimo rancho (i= 1, 2, 3)

$$
\sim \mathrm{N}\left(0, \sigma_{\mathrm{r}}^{2}\right),
$$

$\mathrm{G}=$ Efecto fijo del j-ésimo grupo racial de la vaca $(j=1,2,3,4)$,

$\mathrm{L}_{\mathrm{k}}=$ Efecto fijo del k-ésimo número de lactancia $(\mathrm{k}=$ 1, 2, 3, 4),

$A P_{1}=$ Efecto fijo del l-ésimo año de parto $(I=2000$, 2001, ..., 2004),

$E P_{m}=$ Efecto fijo de la m-ésima estación de parto ( $m=$ seca, lluviosa),

$V(R G)_{n}=$ efecto aleatorio de la n-ésima vaca $(n=$ $1, \ldots, 69)$ anidada en el i-ésimo rancho y el jésimo grupo racial $\sim \operatorname{NID}\left(0, \sigma^{2}\right)$,

$\mathrm{e}_{\mathrm{ijk} \mid \mathrm{mno}}=$ error aleatorio asociado con cada observación NID $\left(0, \sigma^{2}\right)$.

La EPP se estimó con el mismo modelo y únicamente se incluyeron los efectos fijos de la l-ésima época de nacimiento ( $1=$ seca, lluviosa).

\section{EFECTODELAPROPORCIÓNDEGENES}

La información analizada, como se describió anteriormente, se utilizó para estudiar el efecto de la proporción de genes en la PLT, PLD, PLIEP, PLMax, TPLMax, DL, IPC e IEP. Dichas variables se analizaron con el procedimiento MIXED de SAS (SAS, 2002), con el modelo siguiente:

$Y_{i j k l m}=\mu+R_{i}+L_{j}+A P_{k}+E P_{1}+V(R)_{i m}+b_{1}$ $\left(\mathrm{PCTGEU}-\hat{\mu}_{\text {PCTGEU }}\right)+\mathrm{e}_{\mathrm{ijklm}}$

Donde:

$\mathrm{Y}_{\mathrm{ijk} k \mathrm{mn}}=$ Cada una de las variables de respuesta modeladas,

$\mu=$ Media común para todas las observaciones,

$\mathrm{R}_{\mathrm{i}}=$ Efecto fijo del i-ésimo rancho $(\mathrm{i}=1, \ldots, 3)$,

$\mathrm{L}_{\mathrm{i}}=$ Efecto fijo del j-ésimo número de lactancia ( $\mathrm{j}=$ $1, \ldots, 4)$,

$A P_{k}=$ Efecto fijo del k-ésimo año de parto $(k=1, \ldots, 4)$, $E P_{1}=$ Efecto fijo de la l-ésima época de parto $(I=1,2)$,
$\mathrm{V}(\mathrm{R})_{\mathrm{m}}=$ efecto aleatorio de la m-ésima vaca $(\mathrm{n}=$ $1, \ldots, 69)$ anidada en el i-ésimo rancho $\sim \operatorname{NID}(0$, $\sigma^{2}{ }_{v}$,

PCTGEU= Proporción de genes de razas BT como covariable,

$\mathrm{b}_{1}=$ Coeficiente de regresión lineal asociado con la proporción de genes de razas BT,

$\hat{\mu}_{\text {PCTGEU }}=$ Media estimada de la covariable proporción de genes de razas $\mathrm{BT}$, y

$\mathrm{e}_{\mathrm{ijklm}}=$ Error aleatorio asociado con cada observación $\sim \operatorname{NID}\left(0, \sigma^{2}{ }_{\mathrm{e}}\right)$.

El análisis de EPP se realizó de acuerdo a los procedimientos de GLM de SAS (SAS, 2002), con el modelo siguiente:

$$
\begin{aligned}
& \mathrm{Y}_{\mathrm{ijk}}=\mu+\mathrm{R}_{\mathrm{i}}+\mathrm{AN}_{\mathrm{j}}+\mathrm{EN}_{\mathrm{k}}+\mathrm{b}_{1}\left(\mathrm{PCTGEU}-\hat{\mu}_{\text {PCTGEU }}\right) \\
& +e_{i j k}
\end{aligned}
$$

Donde:

$Y_{\mathrm{ijk}}=$ Valor de EPP,

$\mu=$ Media general,

$R_{i}=$ Efecto fijo del $i$-ésimo rancho $(i=1, \ldots, 3)$

$A N_{i}=$ Efecto fijo del $j$-ésimo año de nacimiento $(j=$ $2000, \ldots$ 2004),

$\mathrm{EN}_{\mathrm{k}}=$ Efecto fijo de la k-ésima época de nacimiento de la vaca $(\mathrm{k}=1,2)$,

$b_{1}=$ Coeficiente de regresión lineal asociados con la proporción de genes de razas BT,

PCTGEU = Proporción de genes de razas BT como covariable,

$\hat{\mu}_{\text {PCTGEU }}=$ Media estimada de la covariable proporción de genes de razas $\mathrm{BT}$, y

$e_{i \mathrm{ik}}=$ Error aleatorio asociado con cada observación $\sim \operatorname{NID}\left(0, \sigma^{2}\right)$.

Las medias se estimaron por cuadrados mínimos y las interacciones de primer orden no significativas se removieron del modelo inicial. La medias de efectos principales se compararon con Tukey $(\alpha=0,05)$. Para la covariable PCTGEU se obtuvieron los estimadores insesgados de los coeficientes de regresión. Las soluciones de los efectos fijos de los análisis de varianza se utilizaron para calcular el valor del intercepto de las ecuaciones de regresión; y con éstos se representó la covariable significativa con cada una de las variables de respuesta. 


\section{RESULTADOS}

\section{EfECTOSAMBiENTALES}

Las medias de cuadrados mínimos de PLT, PLD, PLIEP, PLMax, TPLMax, y DL se presentan en la tabla I. El grupo racial únicamente influyó $(p<0,05)$ la PLT y DL, las vacas $3 \mathrm{H} 1 \mathrm{C}$ presentaron los valores más altos de PLT y los demás grupos raciales los más bajos. La DL más larga fue observada en el genotipo 3S1C, intermedias en $3 \mathrm{H} 1 \mathrm{C}$ y $\mathrm{HC}$, y la más corta en SC. Por el contrario, el grupo racial no afectó ( $p>0,05)$ PLD, PLIEP, PLMax, y TPLMax.

El número de lactancia influyó $(\mathrm{p}<0,05)$ la PLT, PLD, PLIEP y PLMax, y no influyó $(p>0,05)$ en TPLMax y DL; los valores más altos para PLT, PLD, PLIEP y PLMax fueron observados en las vacas de cuatro o más lactancias, intermedios en la segunda y tercera, y los más bajos en la primera. El año y la época de parto no tuvieron efectos significativos ( $p>0,05)$ en ninguna de las variables estudiadas, excepto que durante la época seca la PLD fue $8,7 \%$ más alta comparada con la lluviosa.

Las medias de cuadrados mínimos de EPP, IPC e IEP se presentan en la tabla II. El grupo racial influyó significativamente $(\mathrm{p}<0,01)$ la EPP, IPC e IEP; los valores más altos de EPP fueron observados en los animales 3S1C, intermedios en $\mathrm{HC}$ y SC, y los más bajos en $3 \mathrm{H} 1 \mathrm{C}$. Además, las vacas $3 \mathrm{H} 1 \mathrm{C}$ y $3 \mathrm{~S} 1 \mathrm{C}$ presentaron los IPC e IEP más altos, intermedios en SC y los más bajos en HC. El número de lactancia no influyo $(\mathrm{p}>0,01)$ la EPP, IPC e IEP.

\section{EFECTO DELAPROPORCIÓNDEGENES BOS TAURUS}

La proporción de genes BT influyó $(\mathrm{p}<0,05)$ la PLT, TPLMax, DL, e IPC. Las interacciones de primer orden no fueron significativas. La PTL, TPLMax, DL, e IPC incrementaron conforme la proporción de genes BT en los animales aumentó (tabla III). Las vacas con $75 \%$ de genes BT mostraron incrementos de 51,$4 ; 50,8$ y $29,5 \%$ en
PTL, TPLMax y DL, respectivamente, comparativamente con los grupos raciales con $25 \%$ de genes BT (tabla III); el incremento en dichos atributos de PL se asoció con incrementos en los IPC e IEP (tabla III).

\section{DISCUSIÓN}

\section{EFECTOSAMBIENTALES}

Una de las alternativas para incrementar el volumen nacional de leche puede ser el mejoramiento genético de los animales adaptados al trópico en cruzamientos con razas lecheras europeas. Los cruzamientos más comunes se basaron en las razas cebuinas de orígenes diferentes con razas europeas como H y S (Osorio-Arce y Segura-Correa, 2002). El potencial lechero de vacas cruzadas BT X BI es de 1000 a $3800 \mathrm{~kg}$ de leche por lactancia en el trópico mexicano. El alcanzar dicho objetivo es un reto para los productores de los SDP, basado en el conocimiento de que los animales BI tienen lactancias más cortas y se reproducen más lentamente que los BT (González et al., 2006; Lopez et al., 2006). Para superar dicho reto, los SDP requieren de la incorporación de germoplasma para PL en sistemas de cruzamientos (McDowell et al., 1996). La justificación del uso de animales BT es la ventaja de la heterosis proveniente del cruzamiento de animales europeos con razas nativas (Madalena et al., 1990b; Madalena, 1993).

En el presente estudio, las vacas $3 \mathrm{H} 1 \mathrm{C}$ produjeron 12,18 y $26 \%$ más leche por lactancia que $3 \mathrm{C} 1 \mathrm{~S}, \mathrm{HC}$ y $\mathrm{SC}$, respectivamente (tabla I); este hecho puede explicarse parcialmente por dos razones. La primera se relaciona con la diversidad de genes suministrada por los sementales cebuinos, con influencia predominante de Brahman y otros tipos de Cebú desconocidos y que están diseminados en los trópicos del país (Román y Román, 1981; McDowell, 1985), y la segunda, por el ambiente climático y el manejo nutricional propio de cada explotación. Treviño et al. (1980) evaluaron la PL de bovinos Suizos, Holstein y HC. La PLD fue 
de 8,$63 ; 8,66$ y $8,44 \mathrm{~kg}$ para los tres grupos raciales estudiados, respectivamente; este resultado se atribuyó al manejo del ambiente como el pastoreo intensivo, la fertilización y el riego.

Contrario a lo esperado, los animales SC presentaron PL similares a los 3S1C. Dicha respuesta puede deberse al manejo de los animales y a la inclusión de concentrados; sin embargo, no hay una explicación clara de la diferencia. Cunningham y Syrstad (1987) mostraron incrementos importantes en PL e IEP cuando la fracción de genes europeos incrementó hasta $50 \%$; después de este nivel, los incrementos en IEP fueron muy ligeros, y no demostraron una tendencia clara en PL. Los mismos resultados fueron observados en otras regiones tropicales con condiciones similares (Madalena et al., 1990b).

Como un caso único, la PL por lactancia $(4961 \pm 416)$ fue superior en dos o tres veces a los valores de 1322 y 1611 litros de leche en hatos de doble propósito (Hernández et al., 1989). El hecho anterior se explica porque el manejo general de los ranchos ganaderos utilizados en el estudio no es el típico de los trópicos estudiados. En el presente estudio, se incluyen exclusivamente lactancias de 270 días, la PL es el producto de dos ordeños por día, sin apoyo del ternero y la adición de un complemento comercial; mientras que en los SDP tradicionales, la duración de la lactancia es más corta, y el suministro de concentrados no es una práctica común.

De la literatura consultada puede concluirse que las vacas $\mathrm{HC}$ producen más leche que los grupos raciales $\mathrm{SC}, 3 \mathrm{H} 1 \mathrm{C}$ y $3 \mathrm{~S} 1 \mathrm{C}$ en las diversas condiciones tropicales estudiadas (Villegas-Carrasco y RománPonce, 1986; Osorio-Arce y Segura-Correa, 2002). La explicación se basa en la presencia de genes provenientes de razas especializadas como $\mathrm{H}$, asociados con genes de adaptación al ambiente proporcionados por BI. Contrariamente, aquí no se percibió dicha superioridad; la explicación de la inferiori- dad estriba en el mejoramiento del ambiente por la inclusión de complementos concentrados y el manejo del ordeño empleado sin el apoyo de los terneros; que fueron parte del manejo de los animales y no en los estudios anteriores.

La leche producida es el resultado de la conjunción de factores de la vaca, el ambiente y el manejo. De los factores del animal, la edad y la madurez fisiológica influyen en la cantidad de leche cosechada. El número de lactancia influyó positivamente la PL (tabla I). Las vacas de cuatro lactancias fueron superiores en 10, 16 y $30 \%$ comparadas con las de tres, dos y una, respectivamente. La diferencia en el volumen de leche se explica por el crecimiento y desarrollo corporal mayor, conjuntamente con el tamaño de las estructuras intestinales y la glándula mamaria mayores comparado con las vacas más jóvenes (Tucker, 1982). Los resultados obtenidos son similares a los observados previamente por otros investigadores (Hernández-Reyes et al., 2000; ViteCristóbal et al., 2007).

Las condiciones climáticas influyen en la PL cosechada de diferentes formas. Una forma directa es alterando el metabolismo del animal por las temperaturas altas, e indirectamente determinando la estacionalidad de la producción forrajera (Jonsson et al., 1999). El año de parto no influyó en la cantidad de leche obtenida. El hecho anterior se explica parcialmente por la adición de concentrados que minimizó las deficiencias provocadas por el ambiente. La estación de partos representa la conjunción de factores climáticos y el manejo de los animales. La época del año, definida en seca y lluvias, representa la suma de eventos meteorológicos que se expresan en tiempos específicos durante el año. La época del año influyó en la cantidad de leche obtenida por día; la diferencia de $1,0 \mathrm{~kg}$ a favor de la época seca se atribuyó a que en dicha época se presentó un ambiente fresco con temperaturas cercanas a $21^{\circ} \mathrm{C}$ y precipitaciones moderadas que favorecieron un confort metabólico 


\section{REPRODUCCIÓN Y PRODUCCIÓN DE VACAS CON DISTINTO NIVEL DE BOS TAURUS}

apropiado para la PL. Dichos resultados fueron observados en otros estudios de condiciones tropicales diversas (Galavis y Vasquez-Pelaez, 1994; García, 2005).

El efecto del grupo racial, el año y la estación de parto no influyeron la PLD. La pérdida del efecto puede explicarse por las variaciones ambientales imperantes en los sitios estudiados, como fueron interpretados para la PLT anteriormente. Además, la pérdida de efecto del año de parto puede explicarse por el suministro de concentrados, que aparentemente, eliminó algunas diferencias por la insuficiencia del alimento. Los resultados obtenidos son diferentes a los observados en la literatura para SDP en otras condiciones tropicales, donde se de- mostró la superioridad de las vacas HC (Hernández-Reyes et al., 2000; Galavis y Vázquez-Peláez, 1994) en comparación con las SC y 3S1C. En contraste, el número de lactancia influyo la PLD; este efecto se explica parcialmente por las diferencias en edad, por el crecimiento y desarrollo de los órganos asociados con el consumo de alimento, y la partición de nutrientes para formación de leche. Los resultados obtenidos en el presente estudio son apoyados por estudios de Galavis y Vásquez-Peláez (1994), Osorio-Arce y Segura-Correa (2002).

La evaluación de grupos raciales para PL tiene como objetivos optimizar los recursos animales y naturales para alcanzar la rentabilidad de los SDP (Cunningham y

Tabla I. Medias de cuadrados mínimos (土 error estándar) para producción de leche por lactancia (PLT), por día (PLD), por día interparto (PLIEP), al pico de lactancia (PLMax,), días al pico de lactancia (TPLMax) y duración de la lactancia (DL) de bovinos cruzados Bos taurus $x \mathrm{~B}$. indicus en el área tropical de Veracruz, México. (Least square means ( \pm standar error) for lactation milk yield (PLT), milk yield per d (PLD), milk yield per d of calving interval (PLIEP), milk yield at lactation peak (PLMax), days to peak milk yield (TPLMax) and lactation lenght (DL) of crossbreeding Bos taurus $x$ Bos indicus cattle in the tropical area of Veracruz, Mexico).

\begin{tabular}{|c|c|c|c|c|c|c|}
\hline Efecto & PLT (kg) & PLD (kg) & PLIEP (kg) & $\operatorname{PLMax}(\mathrm{kg}) \mathrm{T}$ & TPLMax (días) & DL (días) \\
\hline \multicolumn{7}{|c|}{ Grupo racial ${ }^{1}$} \\
\hline $3 \mathrm{H} 1 \mathrm{C}$ & $4961 \pm 416^{a}$ & $13,5 \pm 0,9^{a}$ & $11,4 \pm 1,0^{\mathrm{a}}$ & $17,1 \pm 1,9^{a}$ & $36,4 \pm 06,5^{\mathrm{a}}$ & $364 \pm 22^{\mathrm{ab}}$ \\
\hline $3 S 1 C$ & $4349 \pm 231^{a b}$ & $11,8 \pm 0,5^{a}$ & $8,8 \pm 0,5^{a}$ & $17,6 \pm 0,8^{a}$ & $40,6 \pm 03,0^{a}$ & $382 \pm 12^{\mathrm{a}}$ \\
\hline $\mathrm{HC}$ & $4070 \pm 238^{a b}$ & $12,7 \pm 0,5^{\mathrm{a}}$ & $9,7 \pm 0,5^{\mathrm{a}}$ & $18,6 \pm 0,8^{a}$ & $31,6 \pm 03,0^{a}$ & $330 \pm 12^{\mathrm{bc}}$ \\
\hline SC & $3676 \pm 175^{a b}$ & $12,0 \pm 0,3^{a}$ & $8,8 \pm 0,4^{a}$ & $18,2 \pm 0,7^{a}$ & $37,1 \pm 02,4^{a}$ & $318 \pm 09^{\mathrm{bcd}}$ \\
\hline \multicolumn{7}{|c|}{$\mathrm{N}^{0}$ de lactancia } \\
\hline 1 & $3067 \pm 276^{b}$ & $10,6 \pm 0,5^{c}$ & $7,4 \pm 0,8^{b}$ & $16,0 \pm 1,1^{b}$ & $36,0 \pm 3,8^{a}$ & $302 \pm 15^{a}$ \\
\hline 2 & $3764 \pm 241^{a b}$ & $11,7 \pm 0,5^{\mathrm{bc}}$ & $9,2 \pm 0,7^{\mathrm{ab}}$ & $16,5 \pm 0,9^{b}$ & $31,9 \pm 3,2^{\mathrm{a}}$ & $318 \pm 13^{a}$ \\
\hline 3 & $4040 \pm 291^{\mathrm{ab}}$ & $12,4 \pm 0,6^{a b}$ & $9,6 \pm 0,7^{\mathrm{ab}}$ & $18,5 \pm 1,1^{1 \mathrm{ab}}$ & $35,0 \pm 3,8^{a}$ & $319 \pm 15^{a}$ \\
\hline 4 & $4475 \pm 259^{a}$ & $13,2 \pm 0,5^{a}$ & $10,6 \pm 0,7^{a}$ & $19,6 \pm 1,0^{a}$ & $35,9 \pm 3,5^{a}$ & $328 \pm 14^{a}$ \\
\hline \multicolumn{7}{|l|}{ Año de parto } \\
\hline 2000 & $3768 \pm 255^{a}$ & $12,2 \pm 0,5^{a}$ & $9,1 \pm$ & $16,9 \pm 0,9^{a}$ & $35,9 \pm 3,3^{a}$ & $308 \pm 14^{a}$ \\
\hline 2001 & $4328 \pm$ & $12,6 \pm$ & $10,3 \pm$ & $19,4=$ & $33,6 \pm$ & $328 \pm 14^{a}$ \\
\hline 2002 & $3474 \pm 266^{a}$ & $11,6 \pm 0,5^{\mathrm{a}}$ & $8,5 \pm 0,5^{\mathrm{a}}$ & $17,1 \pm 1,1^{\mathrm{a}}$ & $33,5 \pm 4,0^{a}$ & $314 \pm 14^{a}$ \\
\hline 2003 & $3775 \pm 275^{a}$ & $11,6 \pm 0,6^{a}$ & $8,9 \pm 1,0^{a}$ & $17,2 \pm 1,0^{a}$ & $35,8 \pm 3,6^{a}$ & $317 \pm 15^{a}$ \\
\hline \multicolumn{7}{|c|}{ Época de parto } \\
\hline Seca & 3921 & 12,5 & 9,6 & 18 & $2,7^{a}$ & $309 \pm 10^{a}$ \\
\hline Lluviosa & $3751 \pm 215^{a}$ & $11,5 \pm 0,5^{b}$ & $8,8 \pm 0,6^{a}$ & $17,4 \pm 1,0^{a}$ & $32,6 \pm 3,3^{a}$ & $325 \pm 11^{\mathrm{a}}$ \\
\hline
\end{tabular}

${ }^{1} 3 \mathrm{H} 1 \mathrm{C}=3 / 4$ Holstein $1 / 4$ Cebú; $3 \mathrm{~S} 1 \mathrm{C}=3 / 4$ Suizo $1 / 4$ Cebú; $\mathrm{HC}=1 / 2$ Holstein $1 / 2$ Cebú; $S C=1 / 2$ Suizo $1 / 2$ Cebú. abcdeDentro de columna y efecto, medias con diferente literal son diferentes ( $p<0,05$; Tukey). 
Syrstad, 1987; Madalena, 1993). Dentro de los componentes del sistema, el manejo eficiente de la reproducción animal permite optimizar la rentabilidad de los mismos. La eficiencia puede estimarse mediante la evaluación de indicadores como EPP, IPC e IEP (Madalena, 1993; Wilson et al., 1998). Por ejemplo, la EPP es el resultado de varios factores asociados con la velocidad de crecimiento, la pubertad, el apareamiento y la naturaleza de los genes (Pérez et al., 1997).

En los SDP, el inicio de la lactancia y la producción de carne son igualmente importantes. Un objetivo puede ser el obtener vaquillas paridas a los 24 meses. Para lograrlo, las vaquillas deben alcanzar la pubertad a los 15 meses de edad. Lo anterior, puede constituirse en un reto para los SDP que usan animales BT x BI. Los animales BI alcanzan la pubertad más tarde y tienen diferencias fisiológicas en crecimiento y productivas con respecto a los BT (Alvarez et al., 2000; Lopez et al., 2006).

Tabla II. Medias de cuadrados mínimos ( \pm error estándar) de la edad al primer parto (EPP, meses), intervalo del parto a la concepción (IPC, días) e intervalo entre partos $x$ Bos indicus en el área tropical de Veracruz, México. (Least square means ( \pm standard error) for age-at-first-calving (d), days open (d), and calving interval (d) of crossbreeding Bos taurus $\mathrm{x}$ Bos indicus cattle in the tropical area of Veracruz, Mexico).

\begin{tabular}{lccc}
\hline Genotipo & EPP & DA & IEP \\
\hline 3 H1C & $33,0 \pm 1,6^{\mathrm{a}}$ & $182,0 \pm 34^{\mathrm{abc}}$ & $455,1 \pm 31^{\mathrm{ac}}$ \\
$3 \mathrm{~S} 1 \mathrm{C}$ & $39,6 \pm 0,6^{\mathrm{c}}$ & $206,1 \pm 16^{\mathrm{c}}$ & $475,6 \pm 16^{\mathrm{abc}}$ \\
HC & $33,2 \pm 0,9^{\mathrm{ab}}$ & $113,4 \pm 15^{\mathrm{a}}$ & $382,7 \pm 16^{\mathrm{a}}$ \\
SC & $34,9 \pm 0,6^{\mathrm{ab}}$ & $137,2 \pm 11^{\mathrm{ab}}$ & $416,0 \pm 12^{\mathrm{ab}}$ \\
\hline
\end{tabular}

${ }^{1} 3 \mathrm{H} 1 \mathrm{C}=3 / 4$ Holstein $1 / 4$ Cebú; $3 \mathrm{~S} 1 \mathrm{C}=3 / 4$ Suizo $1 / 4$ Cebú; HC= $1 / 2$ Holstein $1 / 2$ Cebú; $S C=1 / 2$ Suizo $1 / 2$ Cebú.

EPP: edad primer parto, días; DA: días abiertos, días; IEP: intervalo entre partos, días.

${ }^{a b c}$ Dentro de columna y efecto, medias con diferente literal son diferentes ( $p<0,01$; Tukey).
El análisis de los datos mostró la influencia de los grupos raciales en EPP, DA e IEP (tabla II). Las vacas 3H1C y HC parieron más temprano, y tuvieron DA e IEP más cortos comparativamente que las SC y $3 \mathrm{~S} 1 \mathrm{C}$. La diferencia se atribuyó a la habilidad de crecimiento rápido proporcionado por $\mathrm{H}$, que se expresan por el vigor de adaptación de Cebú al ambiente tropical (Vargas et al., 1998; Gomes y Tewolde, 1999).

Los resultados obtenidos sugieren que la duración del IEP y la PLD tienen una asociación estrecha. La PLD determina el manejo de los DA y el periodo seco. De acuerdo con lo anterior, el IEP de 365 días no es el mejor indicador de PL por día en el hato. Por lo tanto, la PLD durante la lactancia prolongará el IEP, principalmente en aquellas vacas con producciones altas. Los IEP observados en vacas $\mathrm{HC}$ son inferiores a los observados en estudios previos (Darwash et al., 1997; Hernández-Reyes et al., 2001). En resumen, los animales HC tuvieron PL moderadas y EPP, DA e IEP más cortos, lo que sugiere que el mejoramiento del ambiente propicia una expresión más eficiente del comportamiento productivo de los animales con proporción equilibrada de genes europeos y exóticos.

Las vacas $\mathrm{HC}, \mathrm{SC}, 3 \mathrm{H} 1 \mathrm{C}$ y $3 \mathrm{~S} 1 \mathrm{C}$ que consumen forrajes tropicales en condiciones extensivas y complementadas con concentrados con $18 \%$ de PC mostraron comportamientos similares en la PLT, PLD, PLMax, TPLMax y DL. Las PL más altas se observaron en las vacas de tercera lactancia. Los animales HC mostraron mayor habilidad reproductiva que las vacas cruzadas con $\mathrm{S}$. Los resultados observados sugieren la posibilidad de usar los grupos raciales $\mathrm{HC}$ como los más apropiados para PL en el norte de Veracruz.

\section{EFECTODE LAPROPORCIÓNDE GENES BOS TAURUS}

Los estudios de Villegas-Carrasco y Román-Ponce (1986) y Hernández et al. (1989) sugirieron que el grupo racial de la

Archivos de zootecnia vol. 58, núm. 224, p. 690. 
vaca influyó positivamente en la cantidad de leche cosechada por día y lactancia. Se encontró que la covariable proporción de genes BT influyó en la PLT, TPLMax y DL (tabla III); los valores máximos para dichas variables se obtuvieron con animales con $75 \%$ de genes BT; estas diferencias se atribuyen a los efectos complementarios de los genes heredados de las razas parentales. Los animales BT trasmiten a la progenie cruzada la habilidad para aprovechar mejor los nutrientes, mientras que los BI trasmiten información de adaptación al ambiente tropical (Madalena, 1993). Con base en lo anterior, es posible indicar que la proporción de $25 \%$ de genes de BI (B, I y G) fue complementaria en la expresión del potencial para PLT, TPLMax y DL. Adicionalmente, las diferencias observadas entre las proporciones de 25, 50, 62,5 y 75\% de genes Bos taurus no se atribuyeron exclusivamente al grupo genético, sino también a los efectos de selección de $\mathrm{H}$ y S. Dichos resultados sugieren que en las condiciones del estudio, los grupos raciales con $75 \%$ de genes BT fueron los mejores para PL.Cunningham y Syrstad(1987), Rege (1998) y García (2005) observaron que los animales con $62,5 \%$ de genes BT fueron mejores para PL, y también observaron que después de dicho nivel, los incrementos fueron ligeros y sin unas tendencias definidas. Treviño et al. (1980) evaluaron la PL de bovinos $\mathrm{S}, \mathrm{H}^{1} 1 / 2 \mathrm{H} X^{1} / 2 \mathrm{~B}$. La PLD fue de 8,6, 8,7 y 8,4 kg día $\mathrm{a}^{-1}$ para los tres grupos raciales estudiados, respectivamente. La similitud fue explicada por el manejo de las condiciones ambientales incluyendo pastoreo intensivo, suministro de 4,0 kg día${ }^{1}$ de alimento comercial durante el ordeño, y el uso de praderas fertilizadas e irrigadas. Recientemente, Vite-Cristóbal et al. (2007) no observaron diferencias en PLD, PLMax, PLIEP y TPLMax, al evaluar los grupos raciales $1 / 2 \mathrm{BT}$ X $1 / 2 \mathrm{BI}$ y $3 / 4 \mathrm{BT}$ X $1 / 4 \mathrm{BI}$. Lo anterior se atribuyó al mejoramiento del ambiente por la inclusión de suplementos concentrados, doble ordeño y pastoreo rotacional de forrajes nativos.

La DL se explica por los DA, la duración de la gestación y el periodo seco. El resultado observado para DL coincide con los publicados por Madalena et al. (1990a) y Rege (1998) en climas tropicales del mundo, y Hernández-Reyes et al. (2000) y García (2005) en condiciones tropicales de México. Los resultados observados para PLT son apoyados por Holman et al. (1990), quienes concluyeron que los grupos raciales con $75 \%$ de genes BT fueron los más rentables para los SDP.

Tabla III. Relaciones entre características productivas y reproductivas con la proporción de genes de Bos taurus, número de lactancias (n), ecuaciones de regresión, error estándar ( \pm ) de la pendiente y el coeficiente de determinación de bovinos cruzados en el área tropical de Veracruz, México. (Relationships among productive and reproductive characteristics with Bos taurus genes proportion, lactation numbers $(\mathrm{n})$, regression equations, slope standard error $( \pm)$, and determination coefficient of crossbreeding cattle in the tropical area of Veracruz, Mexico).

\begin{tabular}{lcccr}
\hline $\begin{array}{l}\text { Regresión de características productivas } \\
\text { vs. Proporción de genes europeos }\end{array}$ & $\mathrm{n}^{1}$ & Ecuación & $\mathrm{EE}( \pm)$ & $\mathrm{R}^{2}$ \\
\hline${\text { Producción de leche, }\left(\mathrm{kg} \mathrm{vaca}^{-1} \text { lactancia }^{-1}\right)^{2}}^{2}$ & 276 & $\mathrm{Y}=2385,4+33,0 \mathrm{X}$ & 12,5 & 0,81 \\
Periodo del parto al pico de lactancia (días) $^{2}$ & 276 & $\mathrm{Y}=19,6+0,27 \mathrm{X}$ & 0,13 & 0,77 \\
Duración de la lactancia (días) $^{2}$ & 276 & $\mathrm{Y}=248,0+1,72 \mathrm{X}$ & 0,54 & 0,79 \\
Periodo de parto a parto (días) $^{2}$ & 276 & $\mathrm{Y}=330,2+1,78 \mathrm{X}$ & 0,71 & 0,67 \\
Periodo del parto a la concepción (días) & 276 & $\mathrm{Y}=52,5+1,70 \mathrm{X}$ & 0,70 & 0,74 \\
\hline
\end{tabular}

EE: error estándar; ${ }^{1}$ número de lactancias incluidas en el modelo; ${ }^{2}$ Efectos lineales $(p<0,05)$. 
Madalena et al. (1990b) observaron que los SDP con manejos deficientes de animales, el grupo racial con $50 \%$ de genes BT fueron más productivos que las vacas con proporciones menores o superiores de genes BT. En el mismo estudio, Madalena et al. (1990b) concluyeron que cuando el manejo nutricional y sanitario se mejoró, el grupo racial con $50 \%$ de genes BT fue superior a los grupos con proporciones menores únicamente. Lo anterior, se atribuyó a las diferencias existentes en la eficiencia de conversión de los alimentos de los grupos raciales con diferentes proporciones de genes BT.

El IPC es una decisión de manejo que impacta grandemente la eficiencia reproductiva de los hatos tropicales (Ortíz, 1994); mientras que el IEP es un indicador del costo y la ganancia marginal de una vaca en el hato, el cual depende del IPC, duración de la gestación y periodo seco. Los valores máximos de IPC e IEP se obtuvieron con animales de $75 \%$ de genes BT (tabla III). Lo anterior, se relaciona con la habilidad reproductiva de BT (H y S) y por los efectos de la alimentación con forrajes y concentrados. Los valores observados para IPC fueron similares a los publicados por Lozano et al. (1992), mientras que los de IEP fueron similares a los de Hernández-Reyes et al. (2000).

Los resultados observados para el IPC son apoyados por Vite-Cristóbal et al. (2007), quienes encontraron que el grupo racial con $50 \%$ de genes BT fue superior al grupo con $75 \%$ de genes BT; y también observaron que la eficiencia reproductiva de hatos comerciales con $50 \%$ fue superior

\section{BIBLIOGRAFÍA}

Acharya, R.M. 1987. Experiences in crossbreeding in India. In: Dairying in India. P.R. Gupta, editor. Priyadarshini Vihar Press. Delhi. India. p. 27-43.

Alvarez, P.J., J. Spicer, C. Chase Jr., E. Payton, D. Hamilton, E. Stewart, C. Hammond, A. Olson and P. Wetteman. 2000. Ovarian and endocrine characteristics during an oestrous cycle in a los grupos con $25 ; 62,5$ y $75 \%$ de genes BT. La diferencia en días a favor del grupo racial con IEP más corto se explica por la habilidad reproductiva de las vacas $\mathrm{H}$ y $\mathrm{S}$. Los valores observados para IEP indican que el comportamiento reproductivo de los grupos raciales con $25,50,62,5$ y $75 \%$ de genes BT es superior a los grupos evaluados en estudios previos (Vite-Cristóbal et al., 2007).

\section{CONCLUSIÓN}

El mejoramiento en el ambiente propicia una expresión más eficiente de la producción de leche y un comportamiento reproductivo posparto moderado del los animales con $75 \%$ de genes Bos taurus. Las vacas con $75 \%$ de genes Bos taurus en pastoreo rotacional de forrajes tropicales y complementados con concentrado son potencialmente aceptables para producir leche en el ambiente del Norte de Veracruz. El manejo diferencial en la alimentación de los grupos raciales con las mayores proporciones de genes Bos taurus producen respuestas positivas en la producción de leche y el comportamiento reproductivo de los animales.

\section{AGRADECIMIENTOS}

Los autores agradecen al grupo de ganaderos del norte de Veracruz Tenek Federación de Sociedades Cooperativas S.C. de R.L. de C.V., por proporcionar la información pertinente para la realización del presente estudio. El agradecimiento es también extensivo al CONACyT por el apoyo brindado al segundo autor para la realización del estudio.

Angus, Brahman, and Senepol cows in a subtropical environment. J. Anim. Sci., 78: 12911302.

Cunningham, E.P. and O. Syrstad. 1987. Crossbreeding Bos indicus and Bos taurus for milk production in the tropics. FAO Animal Production and Health Paper $n^{\circ}$ 68. Rome. Italy. 89 p. 
Darwash, O.A., G.E. Lamming and J.A. Woolliams. 1997. Estimation of genetic variation in the interval from calving to postpartum ovulation of dairy cows. J. Dairy Sci., 80: 1227-1234.

Galavis, J. and C.G. Vásquez-Peláez. 1994. Estimation of milk production curve in Brown Swiss cattle in the subtropical climate of Mexico. Proceedings $5^{\text {th }}$ World Congress on Genetics Applied to Livestock Production. August 7-12 Guelph, Ontario. Canada. 20: 367-370.

García C., R. 2005. Producción de leche de vacas con diferente porcentaje de genes Bos taurus en el trópico mexicano. Tesis de Maestría. Universidad Autónoma Chapingo. México. $60 \mathrm{p}$.

García, E. 1988. Modificaciones a la clasificación climática de Köppen. Instituto de Geografía. Universidad Nacional Autónoma de México. México, D.F. $276 p$

Gómez, CH. y A. Tewolde. 1999. Parámetros genéticos para producción de leche, evaluación de sementales y caracterización de fincas lecheras en el trópico húmedo de Costa Rica. ALPA, 7: 19-37.

González, S.C., J. Goicochea, M.A. Rodríguez, N. Madrid-Bury y D. González V. 2006. Incorporación al servicio en novillas mestizas doble propósito. ALPA, 14: 1-9.

Hernández, H.V.D., G.A. Ortiz, F.J. Juárez y H. Román-Ponce. 1989. Efecto de la ordeña dos veces al día sobre el comportamiento productivo de ganado cruzado de doble propósito en clima tropical húmedo (Am). En: Resúmenes de la Reunión Nacional de Investigación Pecuaria. México, D.F. p. 191.

Hernández-Reyes, E., V.M. Segura-Correa, J.C. Segura-Correa y M.M. Osorio-Arce. 2000. Intervalo entre partos, duración de la lactancia y producción de leche en un hato de doble propósito en Yucatán, México. Agrociencia, 35: 699705.

Holman, F.W., R.A. Blake, R. Milligan, A.P. Oltenacu, R. Barker, V.M. Hahn and T.R. Rounsaville. 1990. Net margins from different fractions of Holstein genes in Venezuelan herds based on performance estimates by producers and advisors. J. Dairy. Sci., 73: 2952-2964.

ICAR. 1995. Recording guidelines: Appendices to the International Agreement of recording practices. International Committee for Animal Recording. Rome, Italy/RVN, Amhem, the
Netherlands. Disponible en URL: http:// www.icar.org/PDF $\% 20$ files/Rules $\% 20$ and $\%$ 20regulations/Guidelines.PDF.

INEGI. 2005. Anuario estadístico del Estado de Veracruz de Ignacio de la Llave. Disponible en URL: http://www.inegi.gob.mx/est/contenidos/ espanol/sistemas/aee05/estatal/ver/index.htm. Jonsson, N.N., J.W. Fulkerson, M.P. Pepper and M.R. McGowan. 1999. Effect of genetic merit and concentrate feeding on reproduction of grazing dairy cows in a subtropical environment. J. Dairy Sci., 82: 2756-2765.

Lopez, R., M.G. Thomas, D.M. Hallford, D.H. Kiesler, G.A. Silver, B.S. Obeidat, M.D. Garcia and C.R. Krehbiel. 2006. Case study: Metabolic hormone profiles and evaluation of associations of metabolic hormones with body fat and reproductive characteristics of Angus, Brangus, and Brahman heifers. The Profesional Animal Scientist, 22: 273-282.

Lozano, D.R.R., G. Leyva R. y L.A. Moreno F. 1992. Efecto del medio ambiente sobre el comportamiento reproductivo y la fertilidad de vacas de la raza Suizo Americano en el trópico subhúmedo. Téc. Pécu. Méx., 30: 208-222.

Madalena, F.E., M. Lemos A., L. Teodoro R., T. Barbosa R. and B.N. Monteiro J. 1990a. Dairy production and reproduction in Holstein-Friesian and Guzera crosses. J. Dairy Sci., 73: 18721886.

Madalena, F.E., L. Teodoro, R.M. Lemos A., B.N. Monteiro J. and R.T. Barbosa. 1990b. Evaluation of strategies for crossbreeding dairy cattle in Brazil. J. Dairy Sci., 73: 1887-1901.

Madalena, F.E. 1993. La utilización sostenible de hembras F1 en la producción del ganado lechero tropical. Estudio FAO Producción y Sanidad Animal $N^{\circ} 111$. Rome, Italy. $98 \mathrm{p}$.

McDowell, R.E. 1985. Crossbreeding in tropical areas with emphasis on milk, health and fitness. J. Dairy Sci., 68: 2418-2429.

McDowell, R.E., C. Wilk J. and W. Talbott C. 1996. Economic viability of crosses of Bos taurus and Bos indicus for dairying in warm climates. J. Dairy Sci., 79: 1292-1303.

Ortíz, O.A. 1994. Ganado lechero en pastoreo. Instituto Nacional de Investigaciones Forestales y Agropecuarias. Boletín Técnico. № 2 . División Pecuaria. Veracruz. México.

Osorio-Arce, M. and J. Segura-Correa. 2002. 


\section{LÓPEZ-ORDAZ, VITE-CRISTÓBAL, GARCÍA-MUÑIZ, MARTÍNEZ-HERNÁNDEZ}

Reproductive performance of dual-purpose cows in Yucatán, México. Livest. Res. for Rural Dev., 14: 1-9.

Pérez, A., R. Khalil, L. Vaccaro e I. Rodríguez M. 1997. Edad al primer parto y su relación con la producción lechera en vacas de doble propósito. ALPA, 5(Supl. 1): 518-520.

Rege, J.E.O. 1998. Utilization of exotic germplasm for milk production in the tropics. In: Procedings $6^{\text {th }}$ World Congress on Genetics Applied to Livestock Production. January 11-16. Armidale, Australia. 25: 193-201.

Román, P.H. y C. Román. 1981. Producción de leche en sistemas extensivos tradicionales en clima tropical. Téc. Pecu. Méx., 40: 7-15.

SAS®. 2002. User's Guide. Statistics $8^{\text {th }}$ ed. SAS Institute Inc. Cary (NC).

Schaeffer, L.R., E. Minder, C.I. McMillan and B. Burnside. 1977. Nonlinear techniques for predicting 305 days lactation production of Holstein and Jersey. J. Dairy Sci., 60: 16361644.

Sikka, L.C. 1950. A study of lactation as affected by heredity and environment. J. Dairy Res., 17: 231-252.

Treviño, T.R., R. Garza T., J. Monroy L. y C.B. Robles. 1980. Producción de leche en pastoreo rotacional intensivo y semintensivo de Ferrer con vacas Suizo-Pardo, Holstein y cruzas Holstein por Cebú. Téc. Pécu. Méx., 29: 7-11. Tucker, H.A. 1982. Physiological control of mammary growth, lactogenesis, and lactation. Michigan State University Press. East Lansing, Ml.

Vargas, C.A., M. Elzo, C. Chase Jr., J. Chenoweth and A. Olson T. 1998. Estimation of genetics parameters from scrotal circumference, age puberty in heifers, and hip height in Brahman cattle. J. Anim. Sci., 76: 2536-2541.

Villegas-Carrasco, M. del C. y H. Román-Ponce. 1986. Producción de leche durante el proceso de formación de un rancho de doble propósito en el trópico. Téc. Pécu. Méx., 51: 51-61.

Vite-Cristóbal, C., R. López-Ordaz, J.G. GarcíaMuniz, R. Ramírez-Valverde, A. Ruiz-Floresy R. López-Ordaz. 2007. Producción de leche y comportamiento reproductivo de vacas doble propósito que consumen forrajes tropicales y concentrados. Rev. Vet. Méx., 38: 63-69.

Wilson, J.S., S.R. Marion, J.N. Spain, D.E. Spiers, D.H. Keisler and C.M. Lucy 1998. Effect of controlled heat stress on ovarian function of dairy cattle 1. Lactating cows. J. Dairy Sci., 80: 273-285.

Wood P., D.P. 1967. Algebraic model of the lactation curve in cattle. Nature, 216: 164-165. 